\section{Tyrosine kinase inhibitors induced immune thrombocytopenia in chronic myeloid leukemia?}

\author{
Avital F. Barak, ${ }^{1}$ Lilach Bonstein, ${ }^{2}$ \\ Roy Lauterbach, ${ }^{2}$ Elizabeth Naparstek, ${ }^{3}$ \\ Sigal Tavor ${ }^{3}$ \\ 'Internal Medicine Department D, Tel- \\ Aviv Sourasky Medical Center, ${ }^{2}$ Platelet \\ Immunology Laboratory, Rambam \\ Medical Center, Haifa, and ${ }^{3}$ Department \\ of Hematology and BMT, Tel-Aviv \\ Sourasky Medical Center, Israel
}

\section{Abstract}

The outcome and quality of life of chronic myeloid leukemia (CML) patients has remarkably changed with the treatment of tyrosine kinase inhibitors (TKIs). Currently, hematopoietic stem cell transplantation (HSCT) is considered mainly as a third line salvage therapy in cases of TKIs resistance or intolerance. Here we describe a patient with chronic phase CML who developed both resistance and late occurrence of s severe thrombocytopenia on first and second generation TKIs and eventually underwent HSCT. Although the mechanism of the myelosuppression is not fully understood, we showed for the first time the development of dose dependent platelet antibodies in the presence of TKIs, suggesting the possibility of TKIs induced thrombocytopenia. Our case emphasizes that late development of severe myelosuppression during imatinib treatment is probably an important indication for consideration of early HSCT.

\section{Introduction}

Chronic myeloid leukemia (CML) accounts for approximately $20 \%$ of all adult leukemia. It is a clonal myeloproliferative disorder, which is caused by the Philadelphia $(\mathrm{Ph})$ chromosome that produces the constitutively active BCRABL tyrosine kinase. Tyrosine kinase inhibitors (TKIs) act by competitive inhibition of adenosine triphosphate (ATP) that binds to BCR-ABL. This process prevents the downstream signal transduction pathways. ${ }^{1,2}$ Imatinib is the first tyrosine kinase inhibitor to have achieved long term disease control in the majority of patients with CML. ${ }^{2}$ In an internationally randomized study of interferon versus ST1571 (IRIS), $63 \%$ of all imatinib patients' showed a complete cytogenetic response (CCyR) and the estimated event free survival (EFS) was $83 \% .^{3}$ The degree of treatment response at earlier time points in the CML correlates with EFS and time to progression to accelerated phase/ blast crisis (AP/BC).

However, imatinib use is complicated by the development of resistance or intolerance, ${ }^{3-7}$ which leads to suboptimal responses or treatment failure. ${ }^{2}$ Imatinib resistance appears to be multifactorial. ${ }^{8,9}$ The most common mechanisms for the development of this resistance are the occurrence of a mutation in the BCRABL tyrosine kinase domain that inhibits imatinibs ability to bind to ABL and poor patient compliance..$^{9,10}$

Most side effects of imatinib are mild to moderate and are often self limiting. Grade $\geq 3$ neutropenia and thrombocytopenia were reported in $17 \%$ and $9 \%$ of the patients respectively, and most of them occurred within the first two years of imatinib treatment. ${ }^{7}$ Furthermore, most myelosuppression is dosedependent, reversible, and can be managed by dose interruption or reduction. In the IRIS study, $4 \%$ of the imatinib group discontinued therapy owing to an adverse event.

The second generation TKIs - nilotinib or dasatinib - are more potent than imatinib and can overcome most imatinib resistant BCRABL mutations. ${ }^{2}$ In a two year follow up of a phase 2 study, nilotinib was given to patients with chronic phase (CP) or advanced phase CML who had failed, or were unable to tolerate, initial imatinib therapy.Cross-intolerance with imatinib (defined as occurrence of a grade $\geq 3$ adverse event (AE) or persistence or recurrent grade 2 of the same $\mathrm{AE}$ that previously led to discontinuation of imatinib) was observed in a sub-analysis of 121 out of 459 patients with CP and AP. All of the seven patients who discontinued nilotinib did so due to thrombocytopenia. ${ }^{11}$

Additional data suggests that patients who develop severe hematological toxicity with imatinib treatment, are at high risk of having a suboptimal response or hematological crossintolerance with a second generation TKI. ${ }^{12}$

Currently, the general assumption is that an initial trial of second-generation TKIs, before proceeding to hematopoietic stem cell transplantation (HSCT), is reasonable in nearly all patients in whom imatinib treatment fails, particularly, because HSCT is available only to a minority of patients and is associated with significant morbidity and mortality. ${ }^{13}$

The Hammersmith score helps predict the response to second generation TKI's and is based on calculating the cytogenetic response to imatinib, the Sokal high risk group and the recurrence of neutropenia. ${ }^{14}$ It is still unclear whether a small, select group of young CML patients in $\mathrm{CP}$ who fail imatinib treatment should undergo allogeneic HSCT without being first treated with second-line TKIs.

Here we present a CP CML patient who
Correspondence: Avital F. Barak, Internal Medicine Department D, Tel-Aviv Sourasky Medical Center, Tel Aviv, Israel.

Tel: +972.3 .6973313 .

E-mail: ab_brk@yahoo.com

Key words: chronic myeloid leukemia, tyrosine kinase inhibitor, thrombocytopenia, HSCT.

Acknowledgment: the authors thank Dr. Talya Finn for assisting with editing.

Received for publication: 22 August 2011. Accepted for publication: 22 November 2011.

This work is licensed under a Creative Commons Attribution NonCommercial 3.0 License (CC BYNC 3.0).

(C) Copyright A.F. Barak et al., 2011

Licensee PAGEPress, Italy

Hematology Reports 2010; 3:e29

doi:10.4081/hr.2011.e29

developed severe thrombocytopenia during treatment with all three TKI's and we raise the question as to whether an early HSCT should have been performed.

\section{Case Report}

A 34-year-old man was diagnosed with $\mathrm{Ph}$ positive CML in May 2009, after presenting the preceding year with weight loss and a white blood cell count (WBC) of $188 \times 10^{9} / \mathrm{L}$. The patient received hydroxyurea for three weeks followed by imatinib $400 \mathrm{mg} /$ day. Within a month he achieved a complete hematological response (CHR).

The patient entered a clinical study that assessed therapeutic levels of imatinib and he was found to have a sub-therapeutic level of $640 \mathrm{ng}$ (the study estimated an optimal therapeutic level of approximately $1000 \mathrm{ng}) .{ }^{15} \mathrm{After}$ three months of imatinib treatment the BCRABL transcript level decreased by only 0.2 logs as compared to the test performed at diagnosis. In November 2009, the patient complained of flu like symptoms and developed severe thrombocytopenia with a platelet (PLT) count of $22 \times 10^{9} / \mathrm{L}$, WBC of $4.4 \times 10^{9} / \mathrm{L}$ with absolute neutrophil count of $0.8 \times 10^{9} / \mathrm{L}$, and hemoglobin of $12.8 \mathrm{~g} / \mathrm{dL}$. A peripheral blood smear showed atypical lymphocytes with toxic granulation, suggestive of acute viral infection. The following tests were normal or negative; herpes simplex virus, herpes zoster, cytomegalovirus, epstein barr virus, IgM antibodies, hepatitis B surface antigen (HBsAg), hepatitis B surface antibody (HBsAb), hepatitis c virus antibody, antinuclear antibody and human immunodeficiency virus antibody. Bone marrow (BM) aspiration and biopsy revealed a hypoplastic mar- 
row with a decreased number of megakaryocytes, no blast cells and no excess of reticulin. Cytogenetic studies demonstrated 93\% mitotic cells with $\mathrm{Ph}$ chromosome and fluorescent in situ hybridization (FISH) showed $83 \%$ of the cells with BCR-ABL. Mutation analysis did not reveal a mutation in the BCR-ABL. The treatment with imatinib was stopped for two weeks and Nilotinib $400 \mathrm{mg}$ twice daily was started after the platelets rose above 50,000 $\mathrm{LL}$. Within another two weeks, there was a decline in his blood count with PLT $15 \times 10^{9} / \mathrm{L}$, normal levels of WBC $\left(5.6 \times 10^{9} / \mathrm{L}\right)$, ANC of $1 \times 10^{9} / \mathrm{L}$ and hemoglobin of $12.1 \mathrm{~g} / \mathrm{dL}$. Of note, severe thrombocytopenia was also observed with a lower dose of Nilotinib (200 mg twice daily). The treatment with nilotinib was discontinued and the patient was treated empirically with prednisolone $80 \mathrm{mg}$ daily. Within 2 weeks, the PLT level increased to $110,000 \mu \mathrm{L}$. After the treatment failure with nilotinib, dasatinib100 $\mathrm{mg}$ once daily was started, with a similar decrease in PLT level to $21 \times 10^{9} / \mathrm{L}$. At this point, studies to evaluate the possibility of drug (TKI's) induced thrombocytopenia were performed.

Tests for anti-human antibodies IgM, IgG and IgA against PLTs were negative. However, after adding the TKI drugs, drug-dependent antibodies against PLT were found using an immunofluorescence test applying flow cytometry (Figure 1). Moreover, the presence of these antibodies was dose dependent and was thus raised the possibility of drug induced thrombocytopenia.

In August 2010, the status of his disease was again re-evaluated. Cytogenetic studies

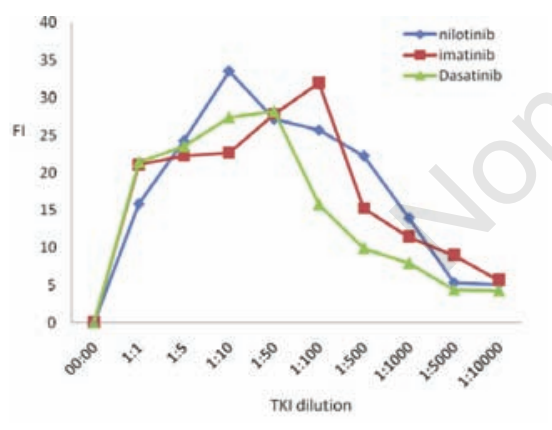

Figure 1. Dose dependent anti platelets antibodies in the presence of tyrosine kinase inhibitors. The presence of antibodies against platelets was detected using platelet immunofluorescence test (PIFT) and the measuring unit was mean fluorescence intensity (FI). Normal group $\mathrm{O}$ platelets from 6 different donors were washed and incubated with patient serum or control serum and with either PBS or TKIs diluted in PBS from 1:1 up to 1:10000. After washing platelets were incubated with FITC labeled goat anti-human IgG and analyzed using flow cytometer. Point zero demonstrates that in the absence of TKIs there are not any antibodies. showed $100 \%$ mitosis with Philadelphia chromosome along with only one log reduction in BCR-ABL load. Bone marrow aspiration and the peripheral counts revealed no blasts thereby implying that the patient was still in the $\mathrm{CP}$ of disease. Since the patient was intolerant to all TKI's, therapy with interferon alpha 3MU three times weekly was started before proceeding to HSCT. During the interferon therapy, there was a minor decrease in PLT to $126 \times 10^{9} / \mathrm{L}$.

In September 2010 the patient underwent an uneventful, allogeneic, peripheral blood stem cell transplantation from a fully matched unrelated male donor after conditioning with BU-CY (busulfan- cyclophosphamide) and standard GVHD prophylaxis. Engraftment was documented on day 13. RT-qPCR for BCR/ABL transcripts performed on day 28 showed $6 \log$ decrease. A repeated test on day 72 showed an increase of BCR-ABL/ABL ratio to $10^{-4}$ and cyclosporine was discontinued. During the following month the patient presented with skin rash, diarrhea and elevated liver enzymes, compatible with acute GVHD. There was a concomitant decrease of BCR-ABL/ABL to $10^{-6}$, 46XY karyotype and 100\% donor chimerism.

Today the patient receives corticosteroids and tacrolimus for chronic limited GVHD. His blood count is normal, with complete donor chimerism and undetectable BCR/ABL.

\section{Discussion}

Our CML patient presented with an interesting combination of resistance and intolerance to TKIs that was manifested mainly as grade 3 thrombocytopenia. Interruption of therapy, dose reduction and therapy with second generation TKIs, did not prevent the recurrence of the adverse hematological events. Mutations in the BCR-ABL tyrosine kinase domain were not found in our patient and he was compliant with medication.

In CML, the hematopoiesis is mainly derived from the $\mathrm{Ph}^{+}$stem cells and myelosuppression is usually observed within the first weeks of TKI treatment. The latter is probably due to delayed recovery of the normal $\mathrm{Ph}^{-}$stem cells. The reduced reserve of residual, healthy $\mathrm{Ph}$ bone marrow (BM) is incapable of reconstituting the peripheral blood count. ${ }^{16,17}$ In the case of our patient, the cytopenia occurred six months after the initiation of imatinib therapy and the $\mathrm{Ph}^{+}$clone was not suppressed, as shown by the $100 \% \mathrm{Ph}^{+}$mitotic cells in the karyotype analysis.

The demonstration of anti-platelets antibodies in the patients' serum only in the presence of TKIs implies that the possible mechanism is indeed drug (TKIs) induced thrombocytopenia.
However, the full significance of this finding is unclear since, unlike in ITP (Immune Thrombocytopenic Purpura), there were decreased numbers of megakaryocytes in the BM, and since no TKI induced immune thrombocytopenia has been previously reported.

HSCT is effective therapy for patients with CP-CML but in the current era of second generation TKI's it is mainly indicated for patients who develop resistance. Less than 5\% of patients develop repeated severe myelosuppression after only a few months of TKI therapy. Late development of severe myelosuppression during imatinib treatment is probably an important indication for consideration of early HSCT. Future studies are needed in order to understand the pathogenesis of recurrent severe myelosuppression during TKI therapy.

\section{References}

1. Copland M. Chronic myelogenous leukemia stem cells: What's new? Curr Hematol Malig Rep 2009;4:66-73.

2. Stein B, Smith BD. Treatment options for patients with chronic myeloid leukemia who are resistant to or unable to tolerate imatinib. Clin Ther 2010;32:804-20.

3. Hochhaus A, O'Brien SG, Guilhot F, et al. Six-year follow-up of patients receiving imatinib for the first-line treatment of chronic myeloid leukemia. Leukemia 2009;23:1054-61.

4. O'Brien SG, Guilhot F, Larson RA, et al. Imatinib compared with interferon and low-dose cytarabine for newly diagnosed chronic-phase chronic myeloid leukemia. N Engl J Med 2003;348:994-1004.

5. DeIninger M, O'Brien SG, Guilhot F, et al. International randomized study of interferon vs ST1571 (IRIS) 8-year follow up: Sustained survival and low risk for progression or events in patients with newly diagnosed chronic myeloid leukemia in chronic phase (CML-CP) treated with imatinib. Blood (ASH anuual meeting abstracts) 2009;114:1126.

6. 0'Brien SG, Guilhot F, Goldman JM, et al. International randomized study of interferon versus ST1571 (IRIS) 7-year followup: Sustained survival, low rate of transformation and increased rate of major molecular response (MMR) in patients (pts) with newly diagnosed chronic myeloid leukemia in chronic phase (CMLCP) treated with imatinib (IM). Blood 2008;112:186.

7. Druker BJ, Guilhot F, O'Brien SG, et al. Five-year follow-up of patients receiving imatinib for chronic myeloid leukemia. $\mathrm{N}$ Engl J Med 2006;355:2408-17.

8. Hochhaus A, Schenk T, Erben P, et al. 
Cause and management of therapy resistance. Best Pract Res Clin Haematol 2009;22:367-79.

9. Weisberg E, Manley PW, Cowan-Jacob SW, et al. Second generation inhibitors of BCRABL for the treatment of imatinib-resistant chronic myeloid leukaemia. Nat Rev Cancer 2007;7:345-56.

10. Branford S, Rudzki Z, Walsh S, et al. Detection of BCR-ABL mutations in patients with CML treated with imatinib is virtually always accompanied by clinical resistance, and mutations in the ATP phosphate-binding loop (P-loop) are associated with a poor prognosis. Blood 2003;102:276-83.

11. Jabbour E, Kantarjian HM, Baccarani M, et al. Minimal cross-intolerance between nilotinib and imatinib in patients with imatinib-intolerant chronic myeloid leukemia in chronic phase (CML-CP) or accelerated phase (CML-AP). Blood (ASH anuual meeting abstracts) 2008;112:3215.

12. Khoury HJ, Mauro MJ, Matloub Y, et al. Dasatinib is well-tolerated and efficacious in imatinib ilntolerant patients with chronic-phase chronic myeloid leukemia (CP-CML). ASH annual meetings and exposition 2009.

13. Gratwohl A, Brand R, Apperley J, et al. Allogeneic hematopoietic stem cell transplantation for chronic myeloid leukemia in Europe 2006: transplant activity, long-term data and current results. An analysis by the Chronic Leukemia Working Party of the European Group for Blood and Marrow Transplantation (EBMT). Haematologica 2006;91:513-21.

14. Milojkovic D, Nicholson E, Apperley JF, et al. Early prediction of success or failure of treatment with second-generation tyrosine kinase inhibitors in patients with chronic myeloid leukemia. Haematologica 2006;95:224-31.

15. Picard S, Titier K, Etienne G, et al. Trough imatinib plasma levels are associated with both cytogenetic and molecular responses to standard-dose imatinib in chronic myeloid leukemia. Blood 2007;109:3496-9.

16. Deininger MW, Goldman JM, Lydon N, Melo JV. The tyrosine kinase inhibitor CGP57148B selectively inhibits the growth of BCR-ABL-positive cells. Blood 1997;90: 3691-8.

17. Druker BJ, Tamura S, Buchdunger E, et al. Effects of a selective inhibitor of the Abl tyrosine kinase on the growth of Bcr-Abl positive cells. Nat Med 1996;2:561-6. 